\title{
Analyse des conditions météorologiques pour la sécurité aérienne à Douala
}

\section{André Lenouo(1), David Monkam ${ }^{(1)}$, Derbetini A. Vondou ${ }^{(2)}$, Roméo S. Tanessong ${ }^{(2)}$ et F. Mkankam Kamga ${ }^{(2)}$}

(1) Département de physique, faculté des sciences, université de Douala BP 24157, Douala, Cameroun

(2) Lamepa, faculté des sciences, université de Yaoundé I, Cameroun lenouo@yahoo.fr

\section{Résumé}

Le 5 mai 2007 à $00 \mathrm{~h}$ 05, heure locale, un Boeing B737-800 de la compagnie Kenya Airways s'est écrasé peu après son décollage de l'aéroport de Douala au Cameroun, faisant 114 morts. Létude statistique montre que le taux d'accidents aériens décroît alors que le nombre de victimes ne décroît pratiquement pas durant ces dernières années dans le monde. Cet accident du vol KQ507 avait lieu alors que les conditions météorologiques étaient mauvaises, dues à un système convectif particulier de type V. Ce mauvais temps est diagnostiqué à l'aide des valeurs d'indices d'instabilités déduits des radiosondages de $12 \mathrm{~h} 00$ UTC et $00 \mathrm{~h} 00$ UTC les 4 et 5 mai 2007 respectivement. L'analyse des champs

D ans la nuit du 4 au 5 mai 2007, un accident d'avion se produit à Douala. Le Boeing B737-800, appartenant à Kenya Airways, s'écrase peu après son décollage de l'aéroport international de Douala le 5 mai à $00 \mathrm{~h} 05$, heure locale. L'épave de l'avion est retrouvée à Mbanga Pongo, un petit village situé à six kilomètres du bout de la piste sud-ouest de l'aéroport de Douala. Ce village très enclavé se trouve dans un marécage de mangroves avec un accès extrêmement difficile. Cela complique l'intervention des secours. Les investigations sur le site du crash indiquent qu'il n'y a aucun survivant parmi les cent quatorze passagers et membres d'équipage.

Le 22 octobre 2005, une autre catastrophe aérienne se produit à Abuja au Nigeria, tuant cent seize personnes. Le 30 janvier 2000, cent soixante-neuf personnes meurent dans un accident d'avion à Abidjan en Côte-d'Ivoire. À chaque fois, le bilan humain est très lourd, on compte près de quatre cents morts pour les trois accidents. Malgré

Figure 1 - Le mont Cameroun, qui culmine à 4095 mètres d'altitude, est à l'origine d'ascendances forcées favorisant des nuages cumuliformes orageux. (C) Normand Roy) 
•.. de certains paramètres météorologiques et l'étude de l'image satellitaire montrent que la situation météorologique était propice au développement de ce type de système en $V$ dans la nuit du 4 au 5 mai 2007 sur le sud du Cameroun. L'étude de l'apport du modèle de prévision numérique ETA à 32 kilomètres de résolution horizontale et 3 heures de résolution temporelle dans la prévision de ce type de phénomène a été faite. Ce modèle pourrait améliorer sensiblement la prévention de telles catastrophes dans la région.

\section{Abstract}

Analysis of weather conditions for the airway security in Douala

On the 5th of May 2007 at 0005 local time, a Kenya Airway's Boeing B737800 crashed a few minutes after take off from the Douala international airport (Cameroon), killing 114 persons. A statistical study shows that, worldwide, in recent years the rate of air crashes has decreased but the numbers of victims increased. Flight KQ507 crashed when meteorological conditions were bad, due to a particular convective system of type $V$. Bad weather was diagnosed using the instability indices computed from the soundings made at 1200 UTC and 0000 UTC 4 and 5 May 2007 respectively. The analysis of the fields of some meteorological parameters and the study of satellite images show that the meteorological situation was favourable to the development of this $V$ type of system during the night of 4 to 5 May 2007 in the South of Cameroon. Evaluation of the contribution of the ETA weather model at 32 kilometres horizontal resolution and time intervals of 3 hours in forecasting this type of phenomenon has been done. It is found that its use in this region could help avoid such catastrophes. les progrès de l'industrie aéronautique, le nombre de victimes des accidents d'avion ne diminue pas. Pour le cas particulier de l'Afrique, il s'y produit environ $25 \%$ des accidents enregistrés annuellement dans le monde pour un trafic d'à peine 4,5\%. La plupart des compagnies aériennes mises sur la liste noire de l'aviation civile internationale sont africaines (Icao, 2008). Les aéroports dans cette partie du monde sont souvent dans un état de délabrement poussé. Les pistes d'atterrissage ne sont pas toujours bien entretenues. Elles côtoient parfois des habitations et la signalisation est souvent inexistante. La sécurité dans les aéroports de dix-sept pays d'Afrique, dont le Cameroun, est assurée par l'Agence pour la sécurité de la navigation aérienne en Afrique et Madagascar (Asecna). Mais la plupart de ces aéroports sont techniquement mal équipés, avec pour beaucoup l'absence de radars. Cependant, la nouvelle génération de B737, équipée d'un radar météorologique, est techniquement évoluée, conforme aux nouvelles exigences du transport commercial. C'était le cas du B737-800 de la Kenya Airways.

En dehors des trois cas ci-dessus cités, divers autres accidents d'avion ont été recensés dans le monde. Mais l'objet de cet article n'est pas de s'intéresser à l'ensemble des catastrophes aériennes ; il tente plutôt d'analyser, en se focalisant sur la nuit du 4 au 5 mai 2007, les conditions météorologiques en relation avec la sécurité aérienne à Douala, ville caractérisée par sa position géographique particulière et une climatologie d'orages et d'activités électriques. Pour situer ce travail dans son contexte, il nous a semblé utile d'approfondir l'analyse statistique des accidents aériens avec les différentes causes et de faire ressortir la contribution de ceux dus aux conditions météorologiques. Il nous a paru aussi nécessaire de calculer certains indices d'instabilité et d'étudier leur relation avec les orages. Nous avons opté pour une étude des indices de convection calculés avant et après le

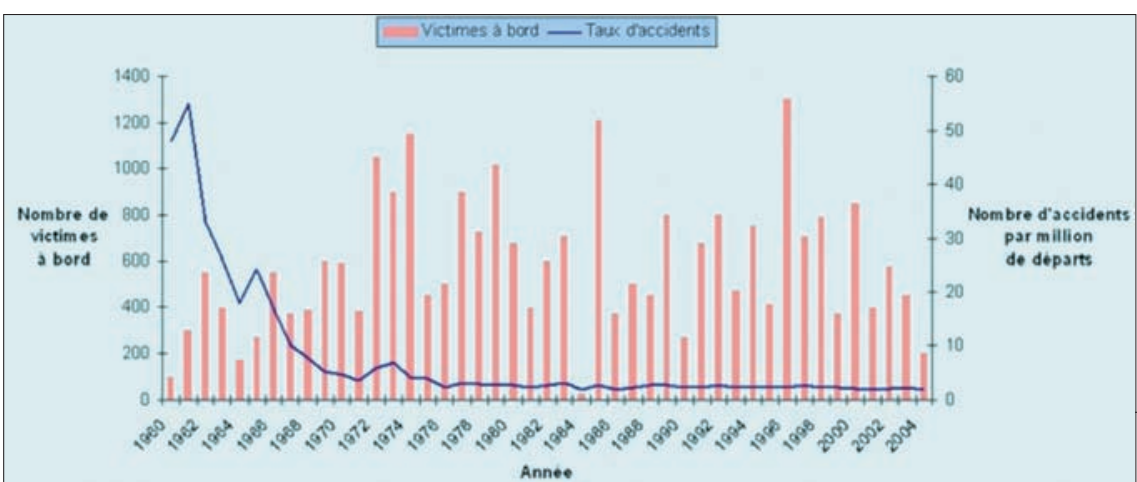

crash à partir des radiosondages de l'aéroport international de Douala. L'étude se concentre sur la région autour de la ville de Douala ; mais, compte tenu de sa situation géographique avec notamment la proximité du mont Cameroun (figure 1), la présence de l'océan Atlantique et l'influence de la mousson africaine, il nous a semblé utile d'examiner la situation synoptique et d'analyser les champs moyens des divers paramètres météorologiques en relation avec les phénomènes pluvio-orageux. Enfin, nous envisageons une perspective d'évolution en prévision numérique pouvant permettre la prévision de ce type de catastrophe.

\section{Analyse des statistiques sur les crashs d'avion}

\section{Croissance du trafic aérien, nombre d'accidents \\ et de victimes}

La catastrophe aérienne du vol numéro KQ507 de Kenya Airways à Douala, le 5 mai 2007, vient augmenter le nombre déjà très élevé des victimes d'accidents d'avion dans le monde malgré les progrès de l'industrie aéronautique. Les statistiques montrent que ces progrès ont contribué à une diminution des accidents aériens, mais le nombre de victimes ne décroît pratiquement pas. Ces statistiques existent pour différents types d'aéronefs. Celles qui sont présentées ici sont tirées de Freissinet (2007) et concernent les avions à réaction de plus de vingt-sept tonnes. Les avions fabriqués dans l'exURSS ne sont pas pris en compte dans cette étude. La figure 2 montre les taux d'accidents et le nombre de victimes à bord, de 1960 à 2004. Au début des années 1960, on compte environ 45 à 55 accidents par million de départs. Ce taux va chuter rapidement pour se stabiliser à moins de 5 accidents par million de départs après 1975. Tandis que le nombre d'accidents par vol ne cesse de décroître, le nombre de victimes, très variable, ne décroît pratiquement pas. Entre 1960 et 1994 (35 ans), on dénombre 20000 victimes, soit en moyenne 571 victimes par an, et entre 1995 et 2004 (10 ans) environ 5700 victimes, soit toujours une moyenne de 570 victimes par an.

Figure 2 - Taux d'accidents et nombre de victimes de 1960 à 2004 (adapté de Freissinet, 2007). 
Figure 3 - Nombre d'heures de vol annuel (NHVA) effectuées et d'avions fabriqués par an de 1970 à 2004 ; les avions fabriqués dans I'ex-URSS n'étant pas pris en compte.

L'évolution des accidents et des victimes est certainement influencée par la croissance du trafic. Si les accidents aériens sont moins probables aujourd'hui qu'il y a vingt ans, il y a aussi beaucoup plus d'avions dans le ciel, et ces avions peuvent transporter plus de personnes. Par conséquent, le nombre de victimes dans le monde ne diminuera pas forcément. Le trafic aérien n'a cessé de croître jusqu'en 2001, année des attentats du World Trade Center. Ces événements dramatiques ont entraîné la faillite de nombreuses compagnies aériennes et une diminution du trafic aérien. Mais, l'aéronautique s'en est vite relevée et, deux ans après, la croissance repartait. La croissance du trafic aérien peut être illustrée en nombre d'heures de vol ou en nombre d'avions fabriqués (figure 3 ). Le nombre d'heures de vol (d'avions fabriqués) est passé de 16 millions d'heures (4 900 avions) en 1970 à 37 millions d'heures (20 000 avions) en 2004.

Le nombre d'avions ne cesse de croître pour satisfaire la demande de transport. L'espace aérien devient donc de plus en plus encombré. Mais, pour l'instant, il n'y a pas de danger de collision car, en même temps, les nouvelles technologies permettent une mesure de plus en plus précise de la position et de l'altitude de l'avion, aidant ainsi à la fois l'équipage et les contrôleurs aériens. Pour accueillir tout le trafic, on a donc réduit les espacements entre avions. Dans les espaces RVSM (Reduced Vertical Separation Minima), les avions se croisent en étant séparés verticalement de 1000 pieds, soit environ 300 mètres.

\section{Quand se produisent les accidents?}

En plus de la croissance du trafic impliquant notamment l'augmentation du nombre d'avions et d'heures de vol, pour mieux cerner l'évolution des accidents et des victimes, il faut répondre à la question «quand se produisent les accidents ? ». Entre le moment où le passager monte dans l'avion et le moment où il en descend, on distingue six phases de vol différentes :

- (a) la phase de roulage où l'avion roule sur le tarmac pour atteindre la piste pour le décollage ou pour atteindre le parking après avoir atterri ;

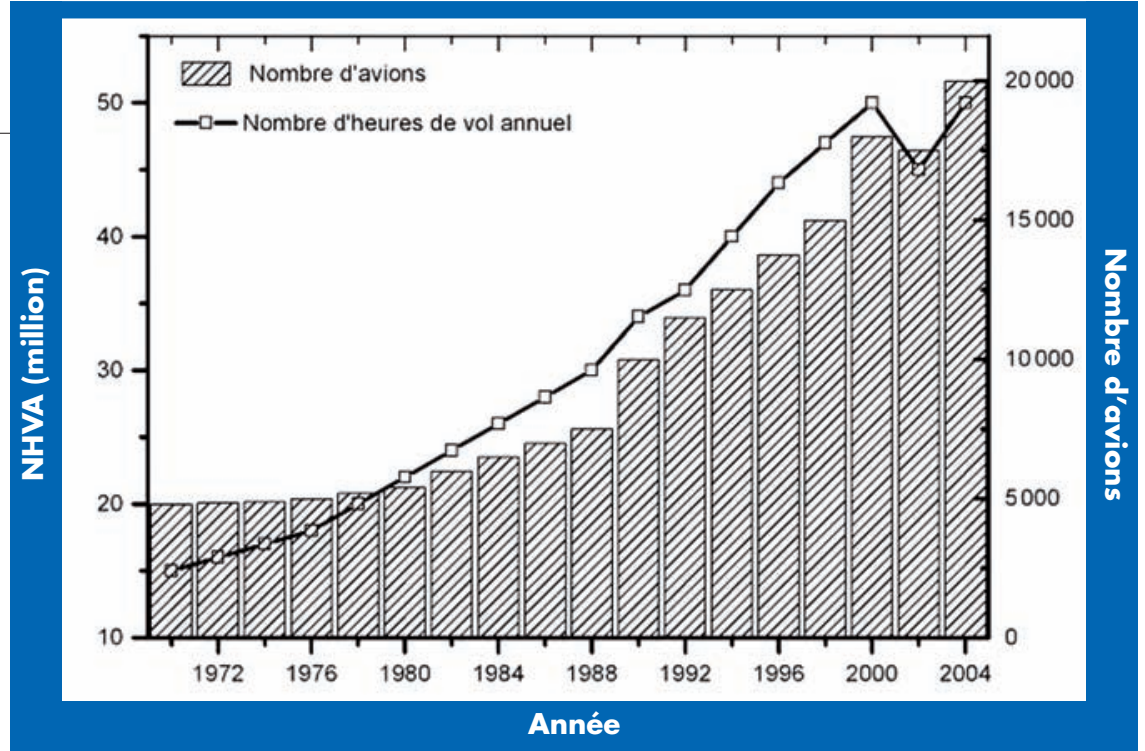

- (b) la phase décollage et montée initiale où, après la mise en puissance de l'avion sur la piste, il quitte le sol et commence à monter ;

- (c) la montée où les becs/volets sont rentrés et l'avion monte jusqu'à son altitude de croisière ;

- (d) la croisière au cours de laquelle l'avion vole à altitude quasi constante et qui correspond généralement à la phase la plus longue d'un vol ;

- (e) la descente et approche initiale où l'avion descend en faisant, si le contrôle lui demande, des boucles en attendant son tour pour amorcer l'approche finale ;

- (f) l'approche finale et atterrissage où l'avion se met en position pour atterrir, puis pose les roues sur le sol et freine.

La figure 4 montre les pourcentages d'accidents et de victimes pour chacune de ces phases de vol. Plus de la moitié des accidents surviennent lors de l'approche finale et l'atterrissage (f). Mais ce n'est pas là où il y a le plus de victimes (une sortie de piste à l'atterrissage ne fera généralement que peu de victimes). Les accidents les plus meurtriers se situent pendant la montée (c). En effet, s'il y a des pannes ou des défauts sur l'avion avant son décollage, c'est principalement là qu'ils vont resurgir et que l'équipage s'en rendra compte. Ainsi, si l'avion doit revenir se poser, il doit engager un demitour, ce qui n'est pas toujours facile, surtout s'il y a déjà des problèmes sur l'avion. La phase de décollage et montée initiale (b) est presque aussi meurtrière que la phase de montée pour un taux d'accidents de moitié plus faible. En règle générale, c'est lorsque l'avion est près du sol que les accidents sont les plus nombreux : la charge de l'équipage est très élevée et la marge de manœuvre pour rattraper une erreur est très faible. L'accident du Boeing 737-800 de Kenya Airways est probablement intervenu dans la phase (b) ou (c).

Figure 4 - Pourcentage d'accidents dans les différentes phases d'un vol pour la période allant de 1995 à 2004 (adapté de Freissinet, 2007)

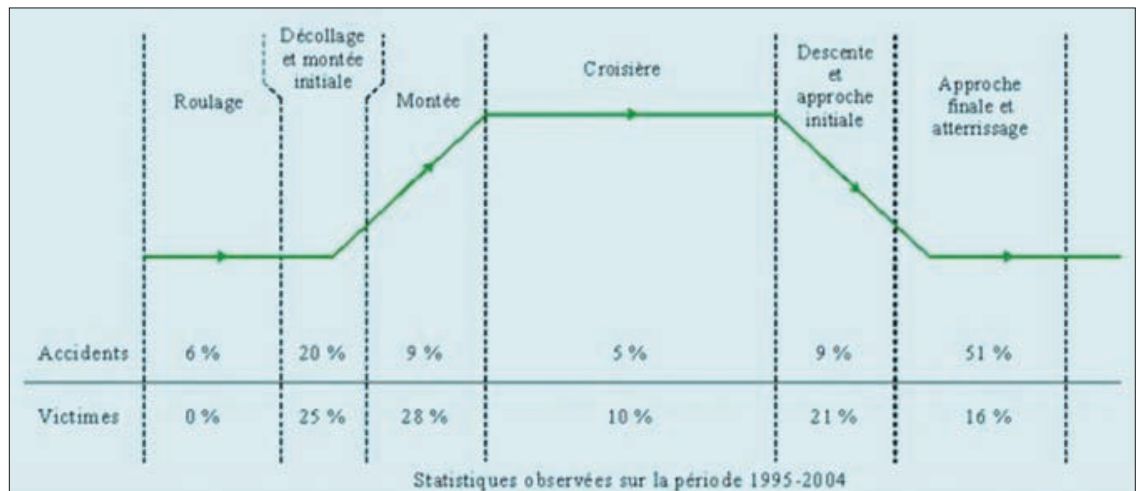




\section{Les principales causes des accidents aériens}

L'énumération des phases de vol avec les taux d'accidents contribue à mieux rechercher les causes. Il est rare qu'un accident aérien soit dû à une cause unique. La plupart des accidents sont la conséquence d'un enchaînement d'événements variés.

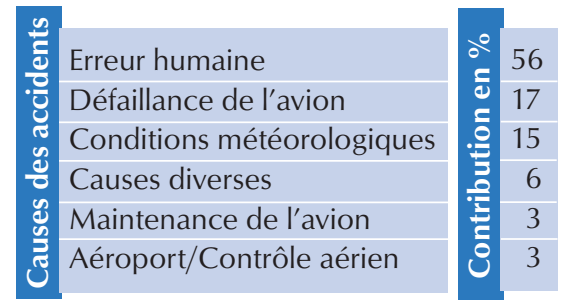

Tableau 1 - Répartition en pourcentage des causes principales dans les accidents aériens (adapté de Freissinet, 2007).

L'erreur humaine est la première cause des accidents d'avion (56\%). Les équipages doivent $s$ 'entraîner régulièrement et intensément pour éviter ces erreurs. En deuxième lieu vient la défaillance de l'avion (17\%), mais cela est de moins en moins vrai avec les avions modernes. Les conditions météorologiques constituent la troisième cause d'accidents avec une contribution de $15 \%$ (cf. tableau 1).

Le présent travail se focalise sur l'étude des conditions climatologiques et météorologiques à Douala, et aborde en détail celles de la nuit du 4 au 5 mai 2007, date à laquelle s'est produit le crash du vol KQ507.

\section{Orages à Douala}

\section{Phénomènes d'orage et de foudre}

Pendant longtemps, les pilotes ont déclaré avoir vu des éclairs dans le ciel au-dessus des nuages sans que personne ne les croie. Mais, ils avaient raison. Les images d'archives prouvent que ces éclairs sont presque invisibles et ne durent qu'une fraction de seconde. Ils mesurent $16 \mathrm{~km}$ de large

Figure 5 - Description d'une cellule nuageuse pouvant donner un orage. Lorsque la taille des gouttelettes d'eau et des cristaux de glace formés dans la partie haute du nuage est trop importante, ils commencent à tomber. Dans leur chute, les gouttes d'eau entraînent de l'air et créent un courant descendant. (Image Météo-France) et s'élèvent à plus de $80 \mathrm{~km}$ au-dessus de l'orage (figure 5). On ignore comment les orages les fabriquent. La formation de la foudre se fait en plusieurs étapes.

\section{Étape 1 : les cumulonimbus}

Le cumulonimbus est un nuage très dense. Il se forme à l'occasion de forts contrastes verticaux de températures, grâce à de puissants courants d'air chaud ascendants. Ces courants entraînent en altitude d'importantes quantités d'eau. Ce nuage peut atteindre une dizaine de kilomètres d'épaisseur. Le cumulonimbus est le nuage le plus craint des aéronautes. Même les plus gros avions de ligne détournent leur route afin d'éviter de les traverser. En effet, la foudre, la grêle et les forts cisaillements de vents à l'intérieur du nuage s'ajoutent au risque de givrage (présent lorsque la température de l'air extérieur est comprise entre -40 et $0{ }^{\circ} \mathrm{C}$ ), et contribuent à mettre en péril l'avion (et ses occupants). Un pilote de planeur ne devrait jamais utiliser les courants ascendants existants dans ou sous ce nuage. Pour lui, il y a deux types de dangers : l'un est lié aux effets de cisaillement entre ascendances et descendances qui peuvent briser le planeur. Le second est plus sournois : les courants ascendants sous un cumulonimbus peuvent atteindre $20 \mathrm{~m} / \mathrm{s}$, être larges et être relativement peu turbulents. Dans ce cas, le planeur est aspiré dans le nuage et le pilote se retrouve en mauvaise posture à cause de la perte des repères visuels. De plus, le phénomène de rafale descendante peut apparaître en dessous du nuage, plaquant l'appareil au sol. Un parachutiste ou parapentiste qui s'engage sous un cumulonimbus prend le risque certain et mortel d'être aspiré rapidement

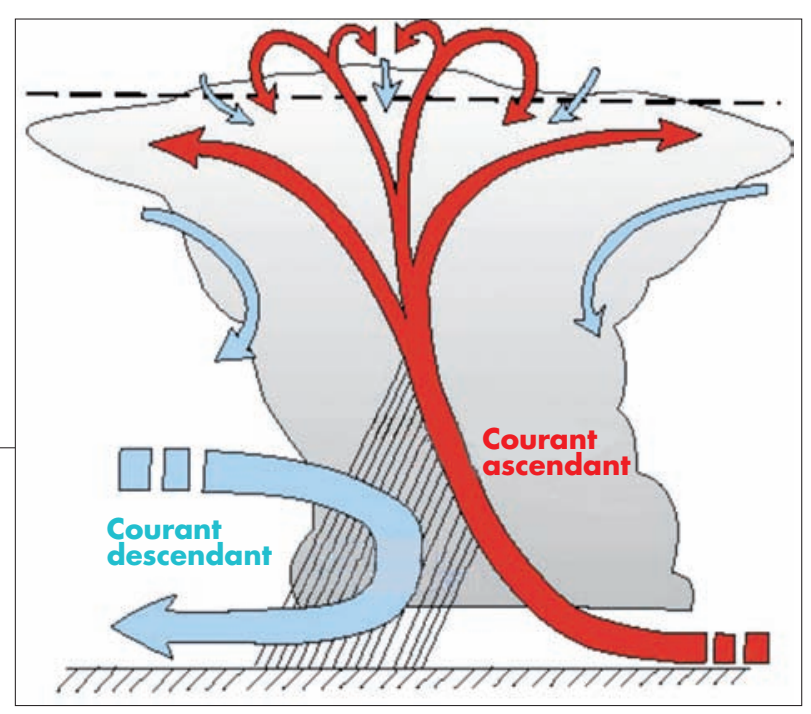

jusqu'au sommet de ce nuage : asphyxié, foudroyé, congelé... S'il en réchappe, il peut avoir des dommages cérébraux irrémédiables suite à un manque d'oxygène.

\section{Étape 2 : les processus d'électrification}

Le brassage qui se déroule à l'intérieur de ces nuages arrache des électrons aux différents éléments d'eau surfondue et de cristaux de glace en suspension. Les frottements engendrés génèrent des charges électriques. Les charges positives s'accumulent au sommet des nuages. À l'inverse, les charges négatives se retrouvent à la partie inférieure. La dissociation des charges dans le nuage orageux génère un champ électrique intense dans l'espace nuage-sol et nuage-nuage. Lorsque son intensité atteint 4 à 10 kilovolts par mètre, une décharge au sol survient entre le sommet et la base du nuage. Une gigantesque étincelle se produit alors, produisant foudre et éclair.

\section{Étape 3 : les éclairs et la foudre}

Les éclairs sont la partie visible de cet amorçage. Ils se développent de deux manières :

- à l'intérieur d'un système orageux (éclairs intra-nuage ou inter-nuages) ;

- entre un nuage et la terre (éclairs nuage-sol).

En moyenne, deux éclairs sur trois sont de type nuage-nuage. C'est ce qui explique que l'on peut entendre gronder le tonnerre sans qu'un impact au sol se produise. La foudre est une très violente et très brève décharge atmosphérique. L'intensité du courant électrique générée varie de 3000 à 300000 ampères. Cette gigantesque étincelle se propage sur plusieurs kilomètres de long. La foudre est un spectacle extraordinaire, mais très dangereux, qui frappe au rythme de 50 à 100 impacts par jour. Nous savons depuis 250 ans que la foudre est un phénomène électrique (Franklin, 1752), même si les scientifiques ne peuvent toujours pas expliquer pourquoi un éclair prend une direction plutôt qu'une autre. Ce n'est 
que depuis peu que nous commençons à comprendre les effets de la foudre sur notre planète.

Des 50000 orages dénombrés par jour dans le monde, les plus violents se produisent en zones tropicales et subtropicales, là où l'humidité est la plus forte et la chaleur la plus intense. C'est le cas de la ville de Douala (figure 6).

\section{Analyse des indicateurs de convection}

La ville de Douala est une agglomération d'environ 2 millions d'habitants. Capitale économique du Cameroun, elle est située sur les côtes du golfe de Guinée, à $9,7^{\circ} \mathrm{E}$ et $4,0^{\circ} \mathrm{N}$ longitude et latitude respectivement. Son climat est très chaud (record maximal de plus de $35^{\circ} \mathrm{C}$ en mai) et très humide avec une pluviométrie de plus de $7001 / \mathrm{m}^{2}$ et vingt jours de pluie en moyenne au mois de septembre (figure 6). Les pluies sont intermittentes, comme cela a été le cas en cette nuit tragique du 4 au 5 mai 2007. Le mois de mai se révèle être le début réel de la période de mousson correspondant à la longue saison de pluie, avec des averses qui peuvent aller jusqu'à une semaine sans interruption, événement localement appelé « la pluie de sept jours ».

Les données utilisées dans cette partie sont des radiosondages réalisés par la division technique de l'Agence pour la sécurité de la navigation aérienne en Afrique et Madagascar (Asecna) à l'aéroport international de Douala. Ces données sont constituées d'une série de deux radiosondages par jour à $12 \mathrm{~h} 00$ et 00 h 00 UTC, conformément au guide de l'Organisation mondiale de la météorologie (OMM), pour les dates du 5 mai 2007 (à 00 h 00 UTC) et 4 mai 2007 ( 12 h 00 UTC). Notons que $00 \mathrm{~h} 00$ UTC et $12 \mathrm{~h} 00$ UTC à Douala et partout au Cameroun correspondent respectivement à $01 \mathrm{~h} 00$ et $13 \mathrm{~h} 00$ heures locales durant toute l'année. La vitesse ascensionnelle du ballon-sonde est d'environ $5-6 \mathrm{~m} / \mathrm{s}$ sur les 1000 premiers mètres. Il faut noter qu'à cause des problèmes de réception du signal radio, deux lancers de ballon ont été effectués le 5 mai 2007 à $00 \mathrm{~h} 00 \mathrm{UTC}$.

Le radiosondage est très utilisé dans les prévisions, sa principale fonction étant la détection d'une atmosphère instable favorable aux développements orageux. Il est très utile dans le domaine de l'aviation et de l'aéronautique. La figure 7 montre les radiosondages réalisés à l'aéroport international de Douala

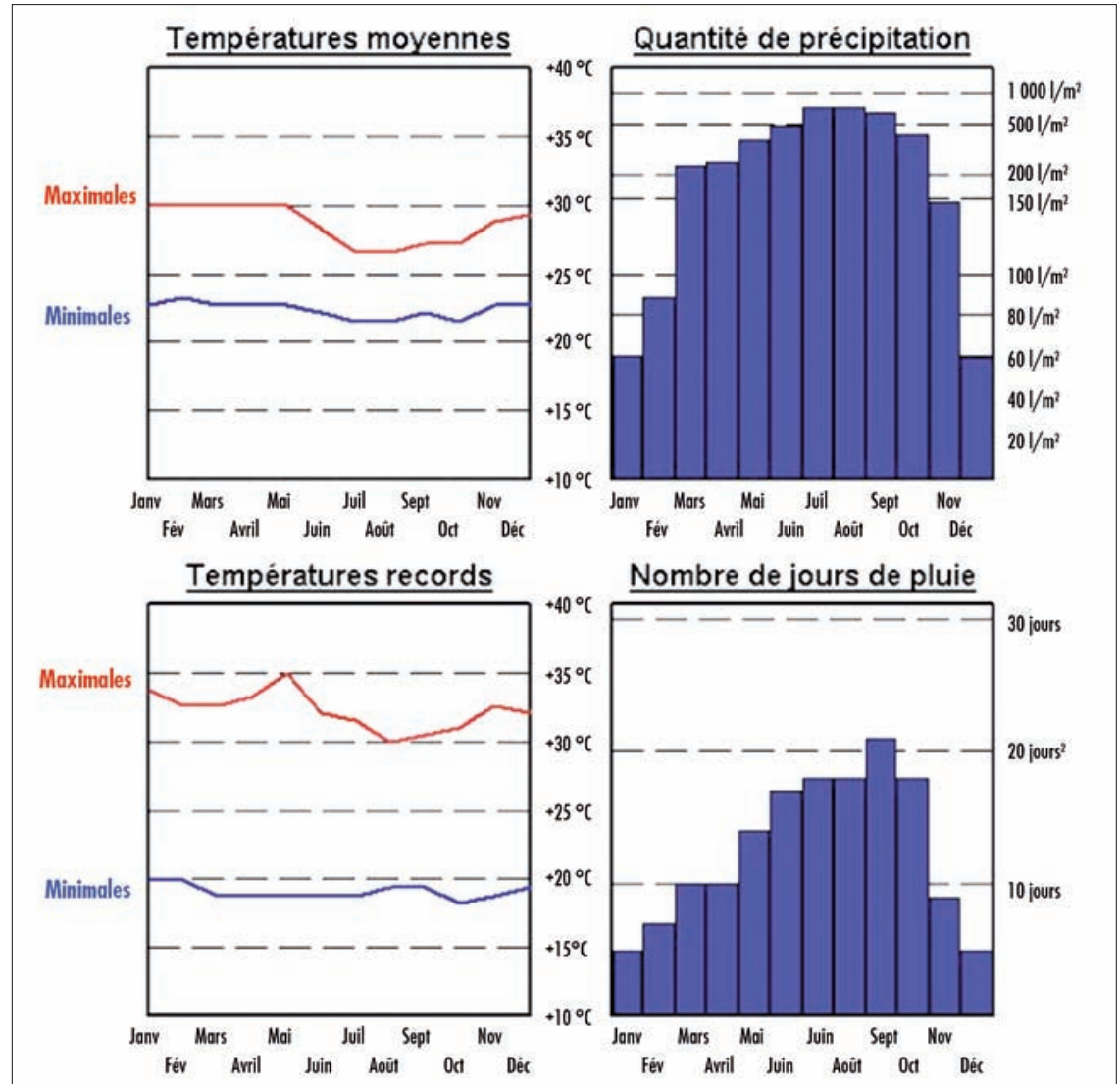

Figure 6 - Variations annuelles de la température

et de la précipitation à Douala $\left(4,0^{\circ} \mathrm{N} ; 9,7^{\circ} \mathrm{E}\right)$. Douala est situé dans le golfe de Guinée, altitude 9 mètres au-dessus du niveau de la mer.

L'hygrométrie est de $80 \%$ en saison sèche et de $99 \%$ en saison des pluies. Ce climat est propice au développement des moustiques et donc au paludisme.

(Image d'après Météo-France)

Figure 7 - Radiosondages du 4 et 5 mai 2007 à $12 \mathrm{~h} 00$ et $00 \mathrm{~h} 00$ UTC respectivement, à Douala. On représente en noir la température du point d'état $\left({ }^{\circ} \mathrm{C}\right)$

en bleu la température du point de rosée $\left({ }^{\circ} \mathrm{C}\right.$ et en trait interrompu rouge

la température pseudo adiabatique potentielle (K).
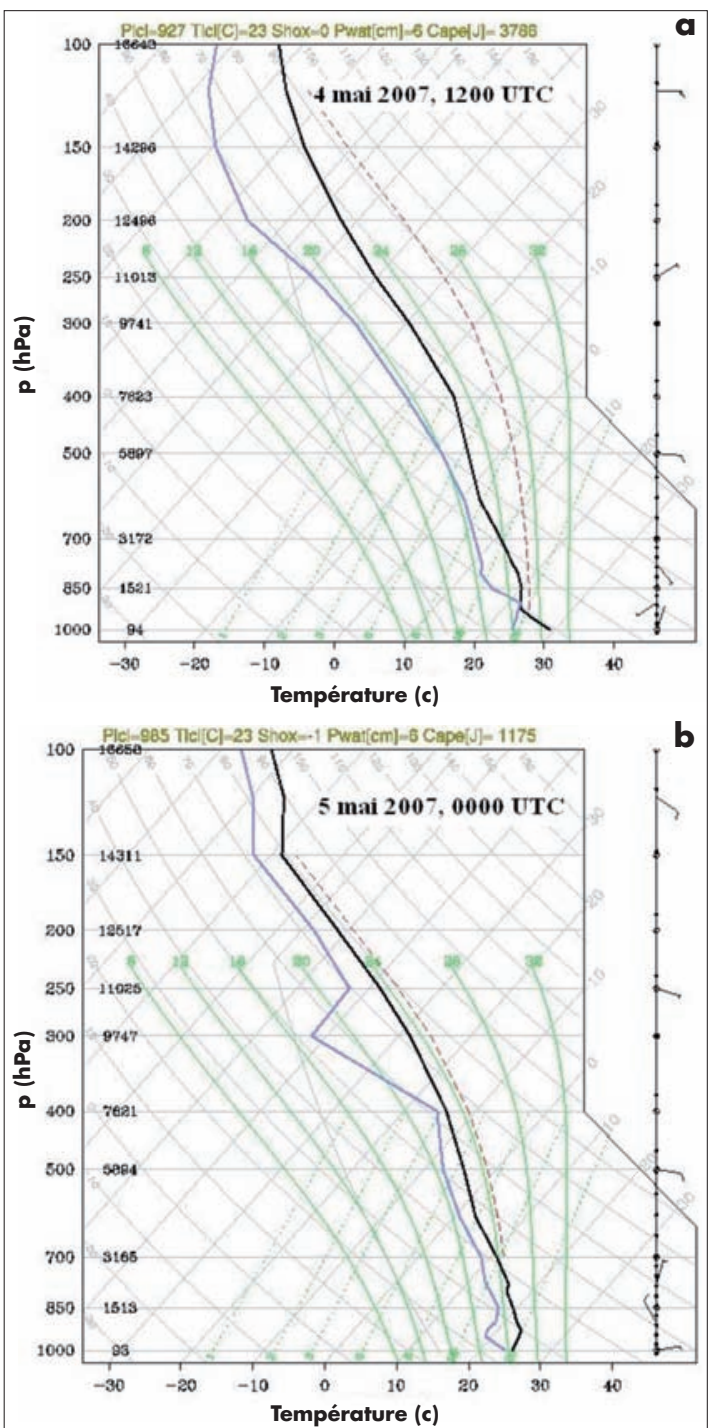
le 4 et le 5 mai 2007 à 12 h 00 UTC et 00 h 00 UTC, respectivement. Diverses grandeurs apparaissent sur cette figure :

- Plcl et Tlcl indiquent la pression et la température du niveau de condensation par soulèvement. Les valeurs de Plcl montrent que le niveau du bas de nuage est à $927 \mathrm{hPa}$ pour le radiosondage du 4 mai et $985 \mathrm{hPa}$ pour celui du 5 mai. La température à ces niveaux reste égale à $23^{\circ} \mathrm{C}$;

- Shox désigne l'indice de Showater. Cet indice est utilisé pour les prévisions des orages compte tenu des différents facteurs intervenant dans l'évolution des cumulonimbus. Les valeurs trouvées sont 0 et -1 pour les deux radiosondages, ce qui indique des averses et/ou des orages probables durant cette période ;

- Pwat désigne la quantité d'eau précipitable. Elle correspond à l'intégration de la vapeur d'eau sur la verticale. Elle est de $6 \mathrm{~cm}$ pour les deux radiosondages. Cette valeur comparée au graphe de précipitation de la figure 6 montre que nous sommes en présence d'un intense épisode orageux ;

- l'énergie potentielle convective disponible ou Convective Available Potential Energy (Cape) est un indice de convection associé à la théorie du soulèvement de la particule d'air. Elle est par ailleurs utilisée comme mesure de l'instabilité convective conditionnelle (Ducrocq et al., 2003). C'est aussi l'indice de puissance des orages et du temps violent (Kerry, 1994). Ainsi, plus la Cape est importante, plus le courant ascendant est puissant et donc plus l'orage disposera de l'énergie. Les valeurs typiques de Cape sont données dans le tableau 2. Les valeurs obtenues pour les deux radiosondages indiquent clairement qu'il y avait une instabilité persistante sur la ville de Douala durant cette période. Car la valeur de Cape d'environ $3786 \mathrm{~J} / \mathrm{kg}$ le 4 mai 2007 à 12 h 00 UTC implique une atmosphère extrêmement instable pouvant conduire à des orages violents et des tornades. Le 5 mai à $00 \mathrm{~h} 00 \mathrm{UTC}$, la Cape vaut $1175 \mathrm{~J} / \mathrm{kg}$, ce qui correspond à une atmosphère modérément instable pouvant donner lieu à des orages modérés ou violents.

Il existe plus d'une soixantaine d'indices d'instabilité dont la plupart sont présentés de manière détaillée par Sénési et Thepenier (1997). Beaucoup de ces indices sont en général calculés à partir de la différence de température entre un niveau des basses couches et un niveau de la moyenne troposphère, avec ou sans soulèvement de la particule. En plus des indices obtenus sur les radiosondages de la figure 7 , nous avons utilisé quatre autres indices :

\begin{tabular}{|l|l|}
\hline Cape $(\mathrm{J} / \mathrm{kg})$ & Stabilité \\
\hline$<300$ & Plutôt stable \\
\hline $300-1000$ & Marginalement instable \\
\hline $1000-2500$ & Modérément instable \\
\hline $2500-3500$ & Très instable \\
\hline $3500-5000+$ & Extrêmement instable \\
\hline
\end{tabular}

Tableau 2 - Les valeurs de Cape et leurs significations en termes de temps sensible.

- la Convective Inhibition (Cin) ou l'énergie nécessaire pour briser le travail de la force de flottabilité ou l'énergie permettant de soulever la particule d'air du sol jusqu'au niveau de la convection libre (Colby, 1984). Cet indice est très utile pour repérer les ascendances violentes au sein d'un nuage cumuliforme. Cette énergie est calculée comme la Cape, mais entre le sol et le niveau de convection libre (Lfc) qui est le niveau d'intersection de la courbe d'état avec la pseudo-adiabatique saturée issue du point de condensation. Or, à Douala, qui est à 9 mètres d'altitude, le niveau de convection libre est très proche du sol, ce qui rend l'utilisation de cet indice non appropriée ;

- l'indice $\mathbf{K}$, qui mesure la probabilité de développement d'orages dans les masses d'air. Sa formule est donnée par la relation :

$\mathrm{K}=\mathrm{T}_{850}-\mathrm{T}_{500}+\mathrm{TD}_{850}-\mathrm{T}_{700}+\mathrm{TD}_{700}$

où $\mathrm{T}$ et $\mathrm{TD}$ désignent respectivement la température et la température du point de rosée aux niveaux 500, 700 et $850 \mathrm{hPa}$. Cet indice vaut 36,7 et 39,1 pour les radiosondages du 4 et 5 mai 2007 à $12 \mathrm{~h} 00 \mathrm{UTC}$ et $00 \mathrm{~h} 00 \mathrm{UTC}$, respectivement. La valeur de l'indice $\mathrm{K}$ durant cette période est supérieure à 35 , ce qui implique un fort risque d'orages avec plus de $80 \%$ de probabilité ;

- l'indice Sweat (Severe Weather Threat) de menace de temps violent est un indice convectif utilisé pour prévoir les orages et les tornades aux États-Unis. La formule de calcul est :

Sweat $=\left(12 * \mathrm{TD}_{850}\right)+(20 * \mathrm{TPO} 2)$ $+\left(2 * \mathrm{SKT}_{850}\right)+\mathrm{SKT}_{500}+$ Shear

Où les termes apparaissant dans cette expression sont définis par :

\section{Signification}

Peu ou pas de convection

Orages de faible intensité

Orages modérés, orages violents possibles Forts orages, tornades possibles Orages violents, tornades probables

Les valeurs calculées de Sweat sont respectivement de 270,4 et 514,4 pour les sondages du 4 et 5 mai 2007 à $12 \mathrm{~h} 00 \mathrm{UTC}$ et $00 \mathrm{~h} 00$ UTC. Ce qui permettait de prévoir une activité orageuse extrêmement faible, voire inexistante le 4 mai à $12 \mathrm{~h} 00$ UTC (Sweat $<300$ ) et des risques d'orages violents et de tornades le 5 mai 2007 à 00 h 00 UTC (Sweat > 500) ;

- le cisaillement du vent : sur les radiosondages (figure 7) apparaissent à droite les indications de la vitesse et de la direction du vent. La vitesse est exprimée en $\mathrm{m} / \mathrm{s}$ (un demi-barbule équivaut à $10 \mathrm{~m} / \mathrm{s}$, un grand à $20 \mathrm{~m} / \mathrm{s}$ ) et la direction selon la position du symbole. Cette donnée étant utilisée notamment pour vérifier s'il existe un cisaillement du vent. Ces deux radiosondages montrent que l'on a dans les basses couches un cisaillement du vent prononcé vers 950 et $800 \mathrm{hPa}$ avec les vents les plus forts atteignant $10 \mathrm{~m} / \mathrm{s}$.

\section{Forçage orographique des basses couches}

La chaîne montagneuse du Cameroun dont le pic le plus haut est d'environ 4100 mètres d'altitude fait partie des chaînes montagneuses en Afrique tropicale (figure 8). D’une façon générale, les filets d'air ont tendance à épouser plus ou moins le profil du relief, de sorte qu'une montagne engendre une zone d'ascendance sur sa face au vent (la mousson dans le cas présent) et une zone de descendance sur sa face sous le vent. La ville de Douala se trouve au pied de la chaîne montagneuse camerounaise (lettre « $\mathrm{C}$ » sur la figure 8 ) sur le versant donnant sur la mer. Sur la figure 9, à $1000 \mathrm{hPa}$, le vent d'ouest
$\mathrm{TD}_{850}$ : température du point de rosée à $850 \mathrm{hPa}$ de pression atmosphérique ; TPO2 : MAX [TPO - 49,0], toutes les températures étant exprimées en ${ }^{\circ} \mathrm{C}$; TPO : index de mesure du potentiel orageux, $\mathrm{TPO}=\mathrm{TD}_{850}+\mathrm{T}_{850}-2 * \mathrm{~T}_{500}$; $\mathrm{SKT}_{850}$ : vitesse du vent en nœuds à $850 \mathrm{hPa}$ de pression atmosphérique ; $\mathrm{SKT}_{500}$ : vitesse du vent en nœuds à $500 \mathrm{hPa}$ de pression atmosphérique ; Shear : $125 *\left[\sin \left(\mathrm{DIR}_{500}-\mathrm{DIR}_{850}\right)+0,2\right]$;

$\mathrm{DIR}_{500}$ : direction du vent en degré à $500 \mathrm{hPa}$ de pression atmosphérique ; $\mathrm{DIR}_{850}$ : direction du vent en degré à $850 \mathrm{hPa}$ de pression atmosphérique. 
Figure 8 - Carte topographique de l'Afrique tropicale. L'intervalle des contours est de $500 \mathrm{~m}$ et les parties teintées indiquent les régions où l'altitude est supérieure à $500 \mathrm{~m}$ par rapport au niveau de la mer.

Les montagnes sont désignées par les lettres : E, montagnes éthiopiennes ; D, montagne du Darfour : C. montagnes camerounaises: $\mathrm{G}$, montagnes guinéennes et $\mathrm{J}$, montagne Jos. (D'après Mekonnen et al 2006)

Figure 9 - Vent (en $\mathrm{m} / \mathrm{s}$ ) et géopotentiel le 4 mai 2007 $18 \mathrm{~h}$ UTC (a et b) et le 5 mai 2007 à $00 \mathrm{~h}$ UTC (c et d) respectivement à $1000 \mathrm{hPa}$ (à intervalles de $10 \mathrm{mgp}$ ) et $300 \mathrm{hPa}$ (à intervalles de $5 \mathrm{mgp}$ ). On peut noter la disparition de la structure en « $\Omega$ entre $18 \mathrm{~h}$ et $00 \mathrm{~h}$ UTC à $300 \mathrm{hPa}$.

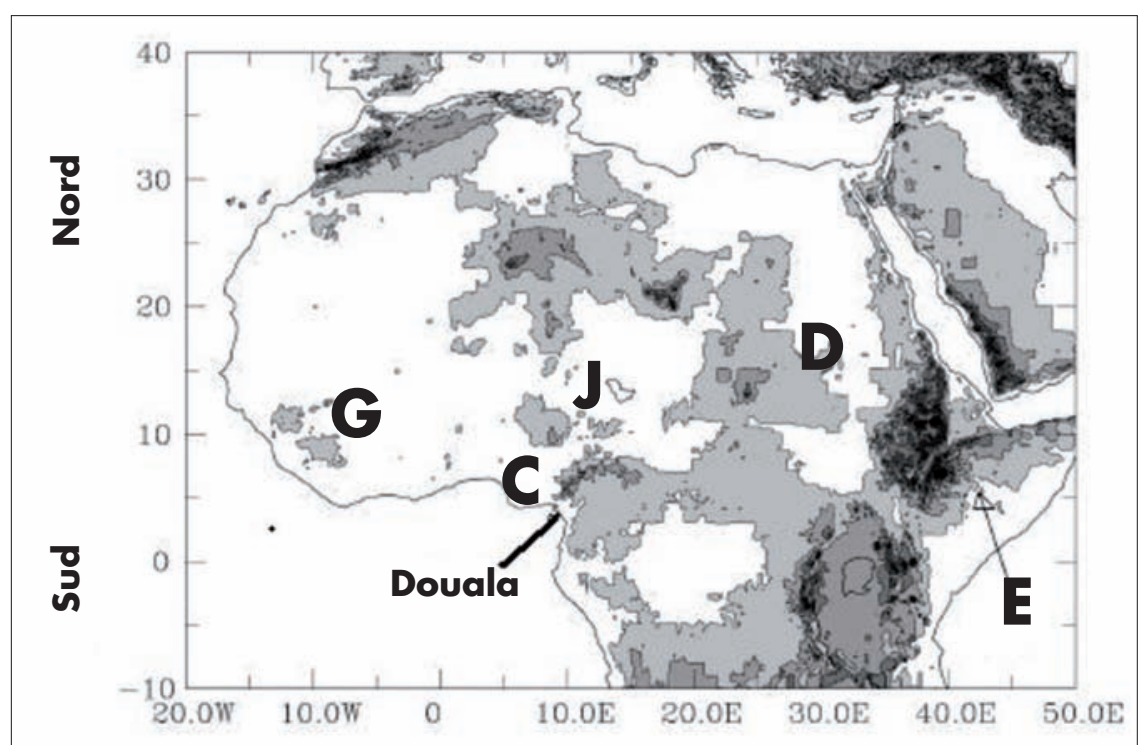

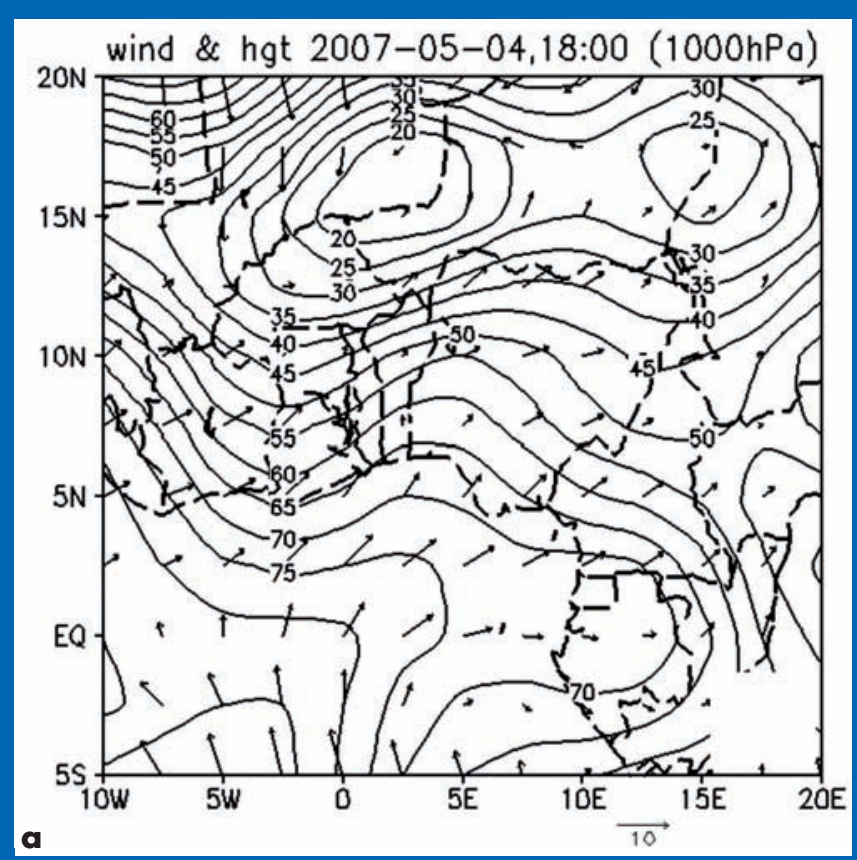

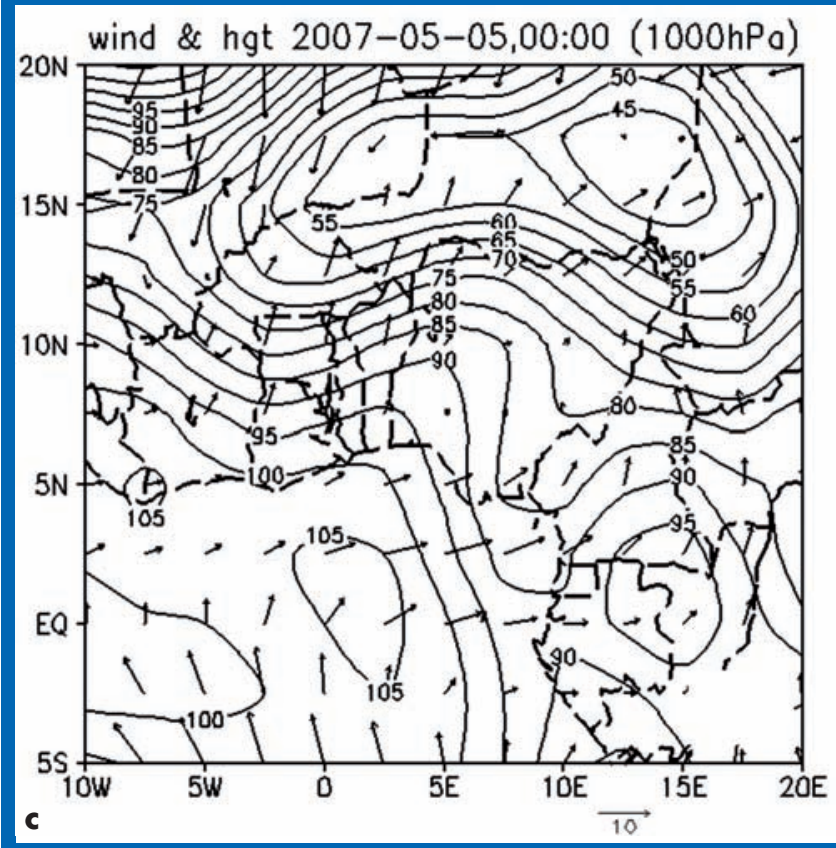

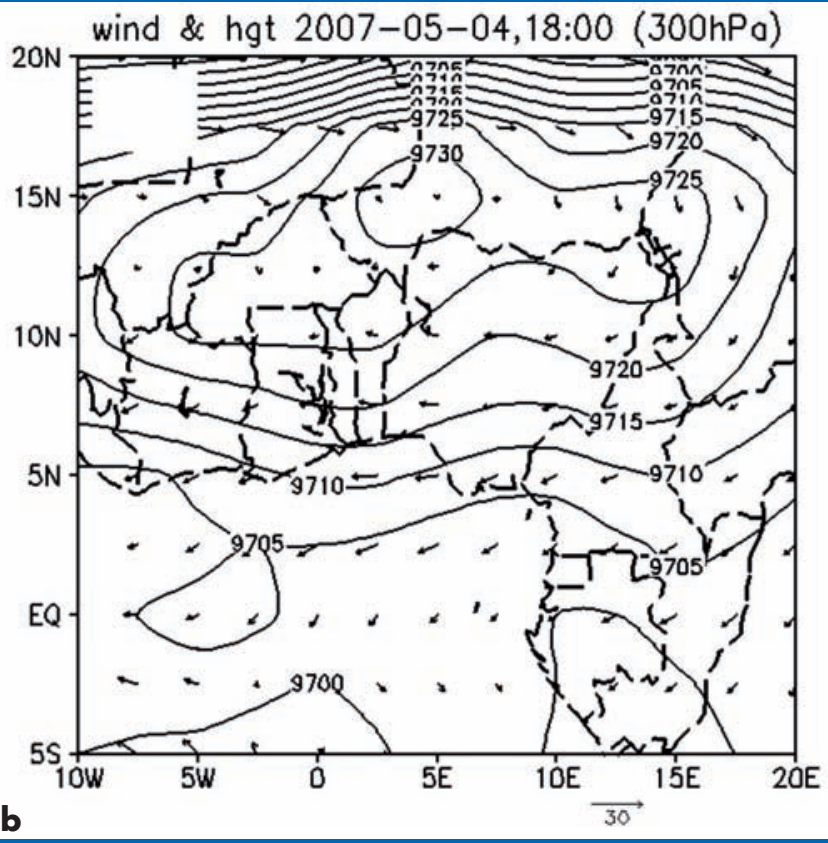

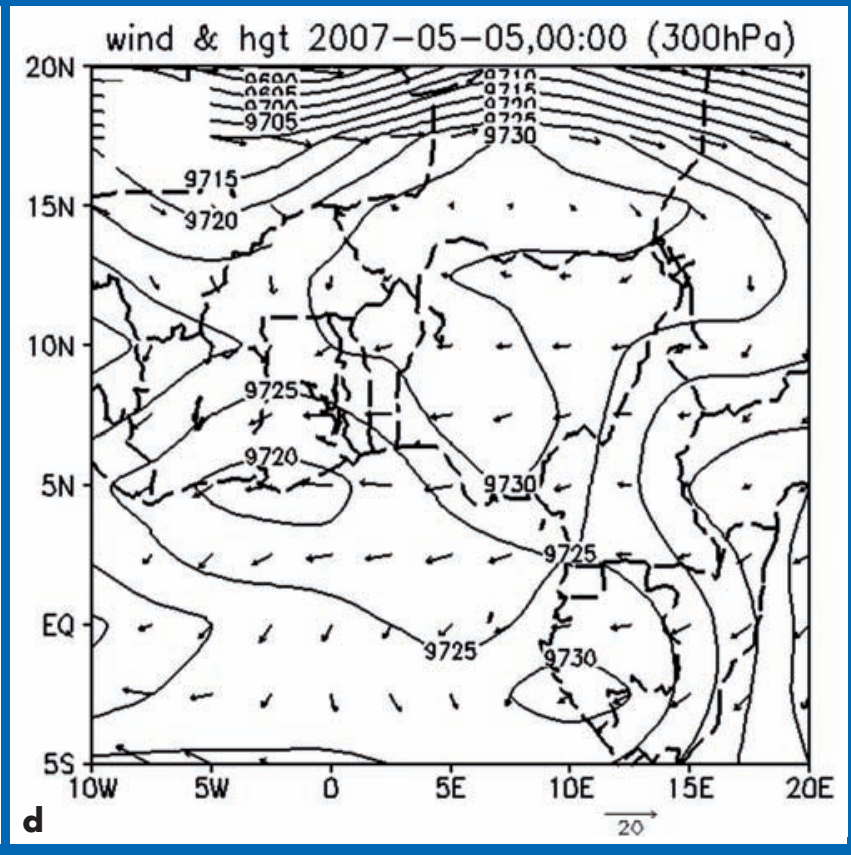


provenant de l'océan Atlantique est fortement dévié sur sa gauche vers la côte camerounaise. Cette chaîne de montagne joue alors le rôle d'une barrière aux courants issus de l'Atlantique, ce qui engendre des mouvements verticaux importants. Or l'air étant convectivement instable, comme l'indiquent les analyses des indices de la convection effectuées sur les radiosondages de la figure 7 , les ascendances forcées par le relief créent des nuages cumuliformes orageux dans cette région. Ces ascendances sont très marquées sur les champs moyens du mouvement vertical à 925 et $500 \mathrm{hPa}$ le 5 mai 2007 à 00 h 00 UTC (figure 10). Ainsi, lorsque le vent humide, issu de l'Atlantique, subit une accélération due aux effets de la chaîne montagneuse, la mousson du sud-ouest arrivant sur la côte du littoral camerounais tend, quant à elle, à être accélérée par la configuration du golfe de Guinée et il s'ensuit finalement une accentuation de la convergence de très basses couches dans la région de Douala.

L'influence de la mer peut, elle aussi, être trouvée dans l'anomalie chaude de température de surface. Avec une température moyenne autour de $30{ }^{\circ} \mathrm{C}$ en surface, on se trouve vraisemblablement en présence d'une mer à température de surface assez élevée. Cette situation où sont conjugués, aux très basses couches de l'atmosphère, la chaleur, le relief, l'humidité et l'instabilité conditionnelle, fait que l'on se trouve dans l'une des régions les plus arrosées au monde. Ainsi, la mesure de la pluviométrie effectuée dans la localité de Debundscha (Limbé), à environ $100 \mathrm{~km}$ de Douala au pied du mont Cameroun, indique que l'on a plus de $10 \mathrm{~m}$ de précipitation par an, avec des pluies quotidiennes sur toute l'année (Derek et Oguntoyinb, 1987).

\section{Analyse de l'image satellitaire}

La figure 11 présente les images satellitaires Météosat à partir de $15 \mathrm{~h} \mathrm{UTC,} \mathrm{le}$ 4 mai 2007, toutes les trois heures. Ainsi, sur ces images du canal IR, les nuages apparaissant en blanc indiquent que les nuages sont d'autant plus intenses qu'ils sont épais. On distingue sur l'image des nuages épais s'étendant sur les côtes de toute l'Afrique de l'Ouest et du golfe de Guinée. Le blanc intense de ces nuages révèle leur fort développement vertical. Seuls les cumulonimbus possèdent ces caractéristiques. Nous sommes donc en présence d'une zone orageuse.

L'extension verticale des cumulonimbus est manifeste et les lignes correspondent à l'extrême sommet du cumulonimbus qui atteint parfois jusqu'à $12 \mathrm{~km}$ d'altitude. En considérant l'extension de la zone orageuse le long de la côte ouest africaine, on note sur l'ensemble de la région du golfe de Guinée la forme caractéristique en V, bien visible à $00 \mathrm{~h} \mathrm{UTC}$, qui est respon- sable de fortes pluies et surtout de cumuls importants du fait de leur stationnarité. Même si cette image présente plusieurs cellules orageuses, les précipitations sont maximales à la pointe du V située dans le golfe de Guinée. Des systèmes en V sont souvent à l'origine des catastrophes, telles qu'au sud de la France les 12 et 13 novembre 1999 (Ducrocq et al., 2003) ou le 3 décembre 2003 dans le golfe du Lion (Hontarrède, 2006).

\section{Les caractéristiques de la circulation}

Les grandeurs météorologiques de la circulation de grande échelle sont issues des analyses opérationnelles de la série de $6 \mathrm{H} \mathrm{du}$ National Centers for Environmental Prediction/National Center for Atmospheric Research (NCEP/NCAR). Cette base de données résulte de l'analyse des paramètres météorologiques observés par un modèle numérique du temps permettant d'interpoler pour les zones non observées (Kalnay et al., 1996). Ainsi, le 4 mai 2007 à $18 \mathrm{~h} \mathrm{UTC}$, à $1000 \mathrm{hPa}$ (figure 9a), on observe une zone de haute pression qui couvre la région Sud-Cameroun en s'inclinant vers le Gabon et le Congo dans la direction sud-est. On note aussi une zone dépressionnaire qui couvre la bande latitude comprise entre $12^{\circ} \mathrm{N}$ et $18^{\circ} \mathrm{N}$. On remarque en altitude $(300 \mathrm{hPa})$ une configuration classique appelée

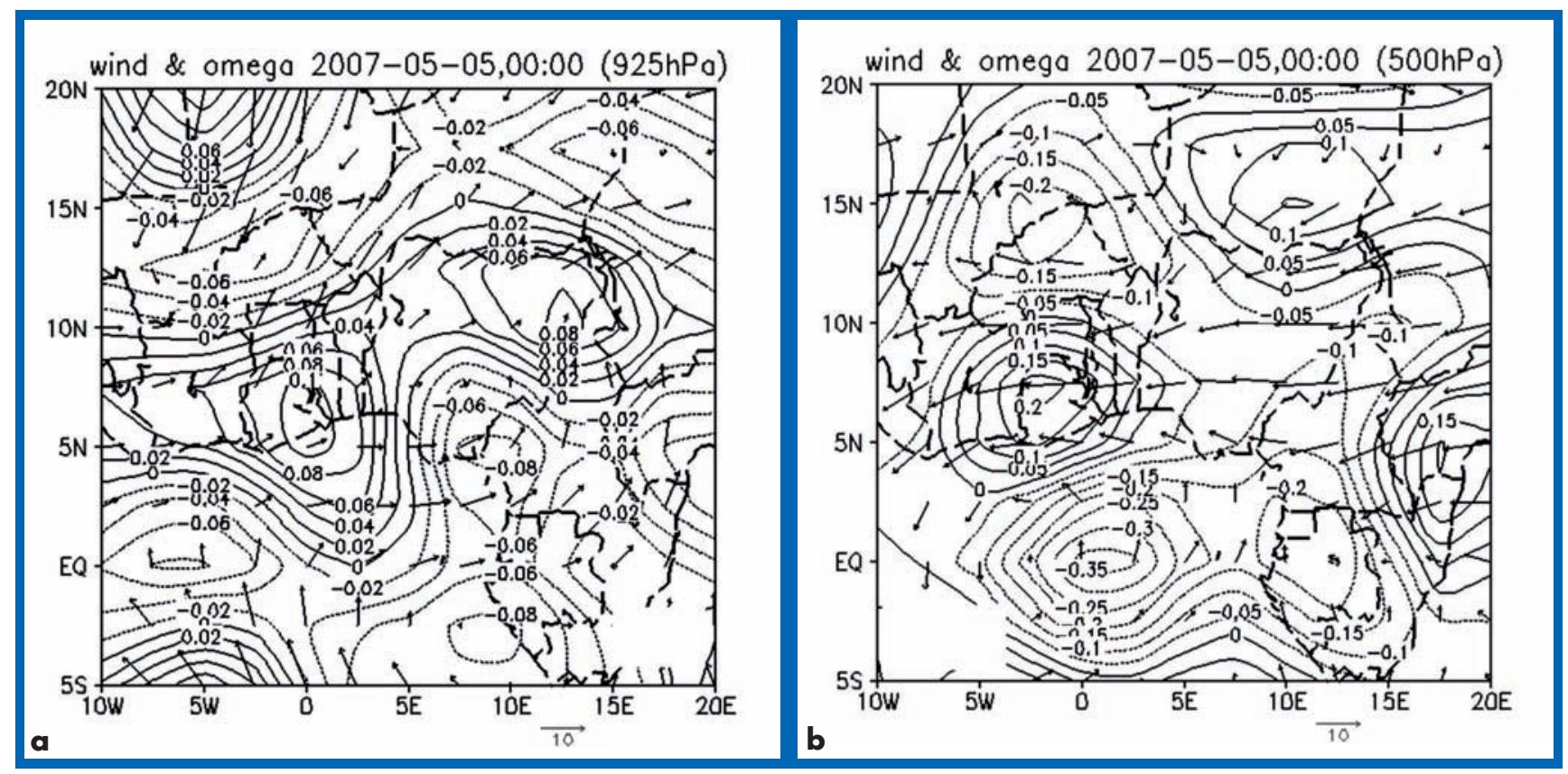



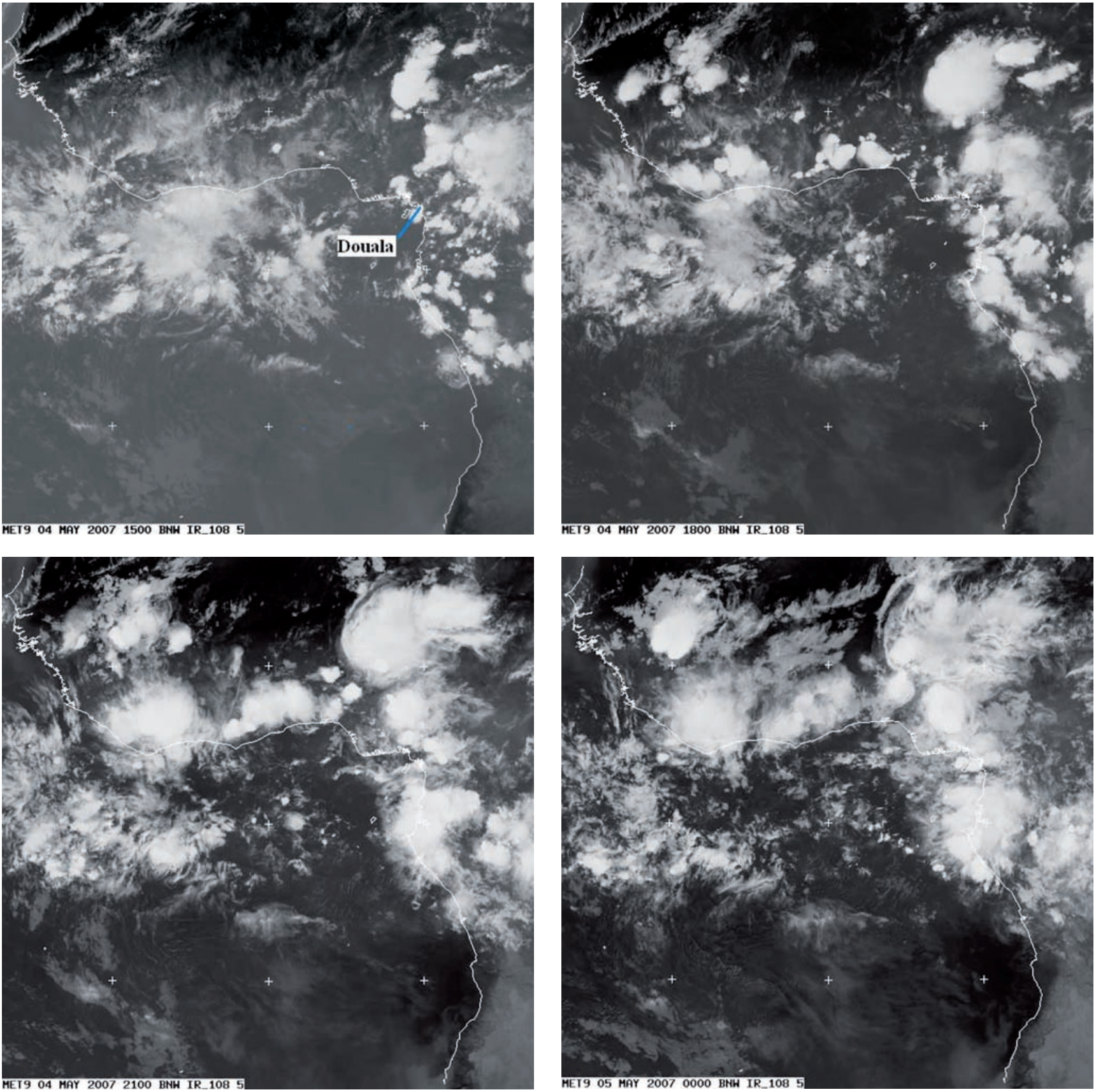

Figure 11 - Images Meteosat-9 sur la bande IR-108 de $15 \mathrm{~h}$ UTC le 4 mai 2007 à $00 \mathrm{~h}$ UTC le 5 mai 2007, toutes les trois heures sur la région couvrant la zone du golfe de Guinée.

situation en $\Omega$, qui est une succession dans la direction SO-NE, alternant anticyclone-dépression-anticyclone, entre les méridiens $10^{\circ} \mathrm{O}$ et $20^{\circ} \mathrm{E}$ (figure $9 \mathrm{~b}$ ). La durée de vie de ce type de circulation atmosphérique de grande échelle en $\Omega$ peut atteindre plusieurs jours. Quelques heures après, ces structures sont complètement désorganisées (figures 9c, 9d), ce qui présage dans la région tropicale des conditions très favorables à un épisode perturbé.

La circulation dans cette région intertropicale est classique. Elle est caractérisée pour cette période de l'année par la circulation de la mousson dans la basse couche de la mer vers le continent. Le 5 mai 2007 à 00 h UTC, elle connaît un renforcement de vent d'environ $10 \mathrm{~m} / \mathrm{s}$ à $1000 \mathrm{hPa}$. À $300 \mathrm{hPa}$, on a un courant de vent d'est plus intense qui s'atténue progressivement lorsqu'il traverse le méridien de Greenwich. Ainsi, durant cette période, la masse d'air qui couvre cette région est potentiellement instable. L'air est relativement chaud, saturé en bas au contact de la mer et est surmonté d'air froid, apporté par les vents d'altitude. Or, la formation d'orage nécessite une impulsion qui imprimera aux basses couches le mouvement ascendant initial. Comme le montrent les figures $10 \mathrm{a}$ et $10 \mathrm{~b}$, on a une ascendance de basses couches atmosphériques dans la région du golfe de Guinée qui persiste jusqu'à $500 \mathrm{hPa}$. Ainsi, cette instabilité de la masse d'air s'amplifie pour renforcer l'orage avec un noyau qui se déplace légèrement de $8,5^{\circ} \mathrm{E}$ à $9^{\circ} \mathrm{E}$ (soit environ $55 \mathrm{~km}$ ) entre ces deux niveaux de pression.

\section{Les conditions associées à l'intensification de la convection}

Pour mettre en évidence les variations brutales des masses d'air qui conduisent aux phénomènes d'orage, on analyse, à partir des données de réanalyse NCEP/NCAR, la température potentielle (figure 12a), la vorticité potentielle 
(figure 12b) et la divergence horizontale (figure 12c). Afin d'apprécier les ascendances et les subsidences subies à l'échelle aérologique par une parcelle d'air surplombant le site, on étudie le comportement de la température potentielle (Iribane et Godson, 1973 ; Yau et Rogers, 1989). La figure 9a montre une masse d'air chaude au niveau de l'équateur qui se refroidit lorsqu'on se rapproche des côtes du golfe de Guinée, avant de se réchauffer sur le continent. Cette variation, aussi faible soit-elle, bouleverse brusquement le temps dans cette région (Triplet et Roche, 1977). Nous avons une confirmation de cette assertion par les variations à $925 \mathrm{hPa}$ de la vorticité et de la divergence horizontale (figures 12b, 12c). Comme l'indique la figure 10, dans la région du golfe de Guinée, on constate une ascendance qui résulte de la convergence horizontale en surface au bas d'une zone dépressionnaire (figure 9c). La vorticité en surface présente de fortes valeurs négatives. Cela indique que l'on a une advection thermique chaude qui tend à renforcer l'ascendance.

\section{Prévision à posteriori de l'épisode orageux par le modèle ETA}

La prévision numérique du temps a été développée comme l'un des outils pour tenter de prédire l'évolution du temps et en particulier les événements extrêmes. En effet, la prévision, plusieurs heures à l'avance d'un événement, permet de prendre des mesures adéquates pour éviter ou atténuer les dommages tels que les dégâts matériels et pertes en vies humaines. Le modèle ETA a été initialement développé en Yougoslavie (Mesinger,
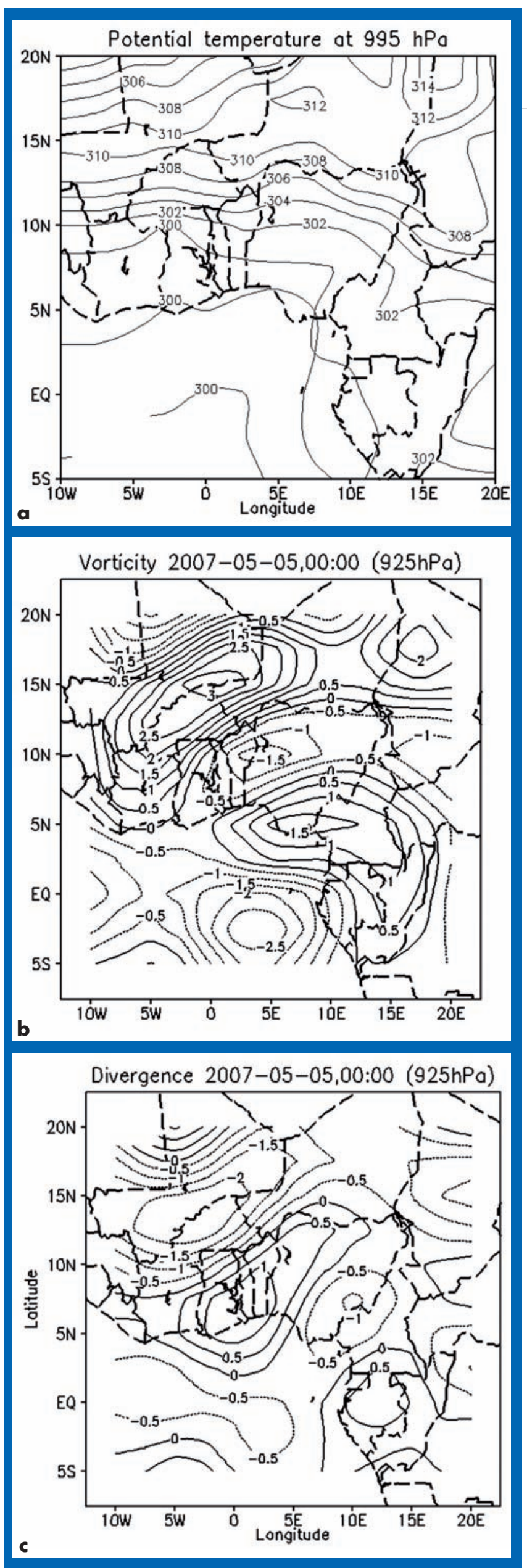

Figure 12 - Température potentielle en $\mathrm{K}$ (a) à $995 \mathrm{hPa}$,

vorticité potentielle (b) et divergence horizontale (c) en $10^{-5} \mathrm{~s}^{-1}$ à $925 \mathrm{hPa}$ le 5 mai 2007 à 00 h UTC.

1984), puis aux États-Unis au NCEP (Rogers, 1995) où il est encore utilisé de façon opérationnelle. En zone tropicale, il a été utilisé sur 1'Amérique notamment par Fennessy et Shukla (2000), et nous l'avons choisi pour faire à posteriori une prévision sur 48 heures avec une résolution spatiale de $32 \mathrm{~km}$. Le schéma de convection de Kain-Fritsh (Kain et Fritsh, 1993) a été utilisé, car le réajustement unidimensionnel qu'il propose s'appuie sur la conservation du flux de masse et prend en compte à la fois les mouvements ascendants et descendants dans une parcelle d'air, phénomènes observables dans la région du golfe de Guinée. Le modèle est initialisé à $00 \mathrm{~h}$ UTC le 4 mai 2007 par les données NCEP du Global Forecasting System (GFS), qui fournit aussi les conditions aux limites du modèle ETA toutes les six heures. Les données du satellite TRMM (Tropical Rainfall Measuring Mission), spécialement équipé pour la mesure des précipitations en zones tropicales et subtropicales, ont été utilisées pour vérifier les prévisions obtenues par le modèle ETA.

\section{Prévision des caractéristiques de la circulation générale}

La figure 13 présente une succession de prévisions du géopotentiel à $300 \mathrm{hPa}$ issue du modèle ETA, par pas de trois heures le 4 mai 2007, à partir de 18 h UTC, superposée à la pression au niveau de la mer durant cette même période afin de pouvoir mettre en évidence des liens entre les champs en surface et en haute atmosphère. La haute pression en surface (entre 1012 et $1008 \mathrm{hPa}$ ) est permanente dans la région 
du golfe de Guinée avant de décroître dans la direction nord-est sur le continent. On peut noter l'anticyclone en basse altitude autour du golfe de Guinée qui se renforce entre $18 \mathrm{~h}$ UTC le 4 mai 2007 et 00 h UTC le 5 mai 2007, sûrement à cause du flux d'ouest. On note aussi la persistance d'une dépression vers le nord du lac Tchad qui s'accompagne d'une baisse de la pression au niveau de la mer. En altitude, on observe plutôt un phénomène inverse durant la même période, dû au flux d'est à ce niveau. Si la dépression dans la région du golfe de Guinée perdure, l'anticyclone se déplace d'abord moyennement entre $18 \mathrm{~h}$ et $21 \mathrm{~h}$ UTC, puis très rapidement vers l'ouest entre $21 \mathrm{~h}$ et $24 \mathrm{~h}$ UTC, tout en restant dans les $10^{\circ}-15^{\circ}$ de latitude Nord. Cette situation permanente de haute pression en surface et de dépression en altitude dans la zone du golfe de Guinée est propice au développement des perturbations orageuses de cette partie du globe.

\section{Prévision des précipitations et comparaison avec les données de TRMM}

La figure 14 présente les précipitations totales au sol prévues par le modèle ETA. Nous observons une vaste zone de perturbation dans le golfe de Guinée et sur l'ensemble des côtes ouest-africaines. Ces précipitations résulteraient des interactions complexes entre le continent et l'océan conduisant à une forte activité convective de la mousson dans cette région. Le modèle ETA réussit à détecter les précipitations autour de la

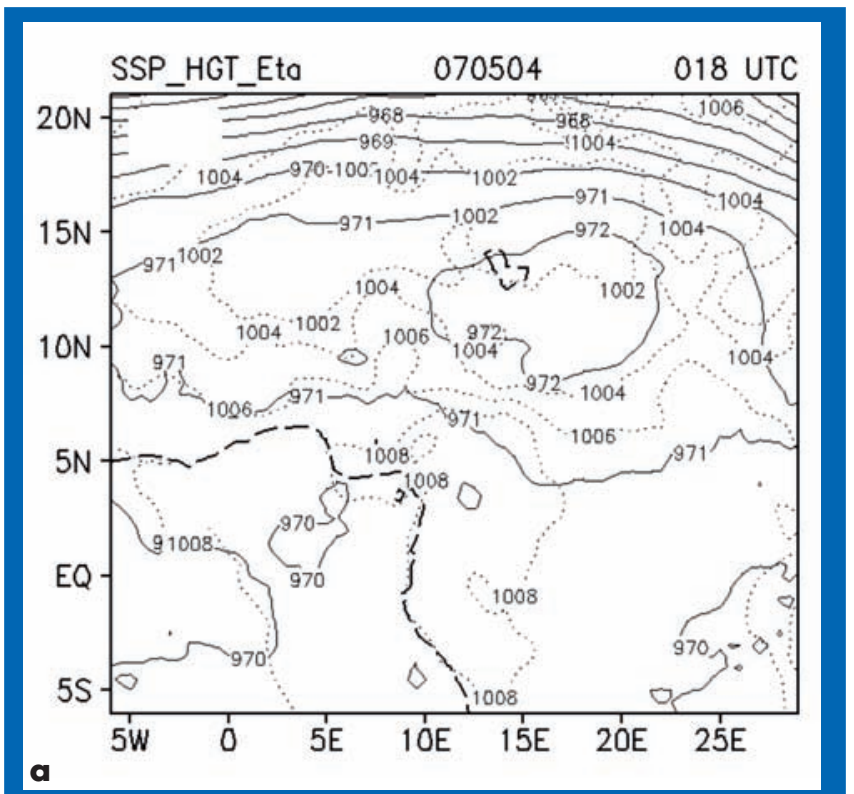

Figure 13 - Prévision du modèle ETA du 4 mai 2007 à partir de $18 \mathrm{~h}$ UTC, par pas de trois heures : (a) $18 \mathrm{~h}$ (b) $21 \mathrm{~h}$ et (c) $24 \mathrm{~h}$ le 4 mai 2007 du géopotentiel à $300 \mathrm{hPa}$ à intervalles de 1 dam (en trait continu) superposé au champ de pression au niveau de la mer à intervalles de $2 \mathrm{hPa}$ (en pointillé).
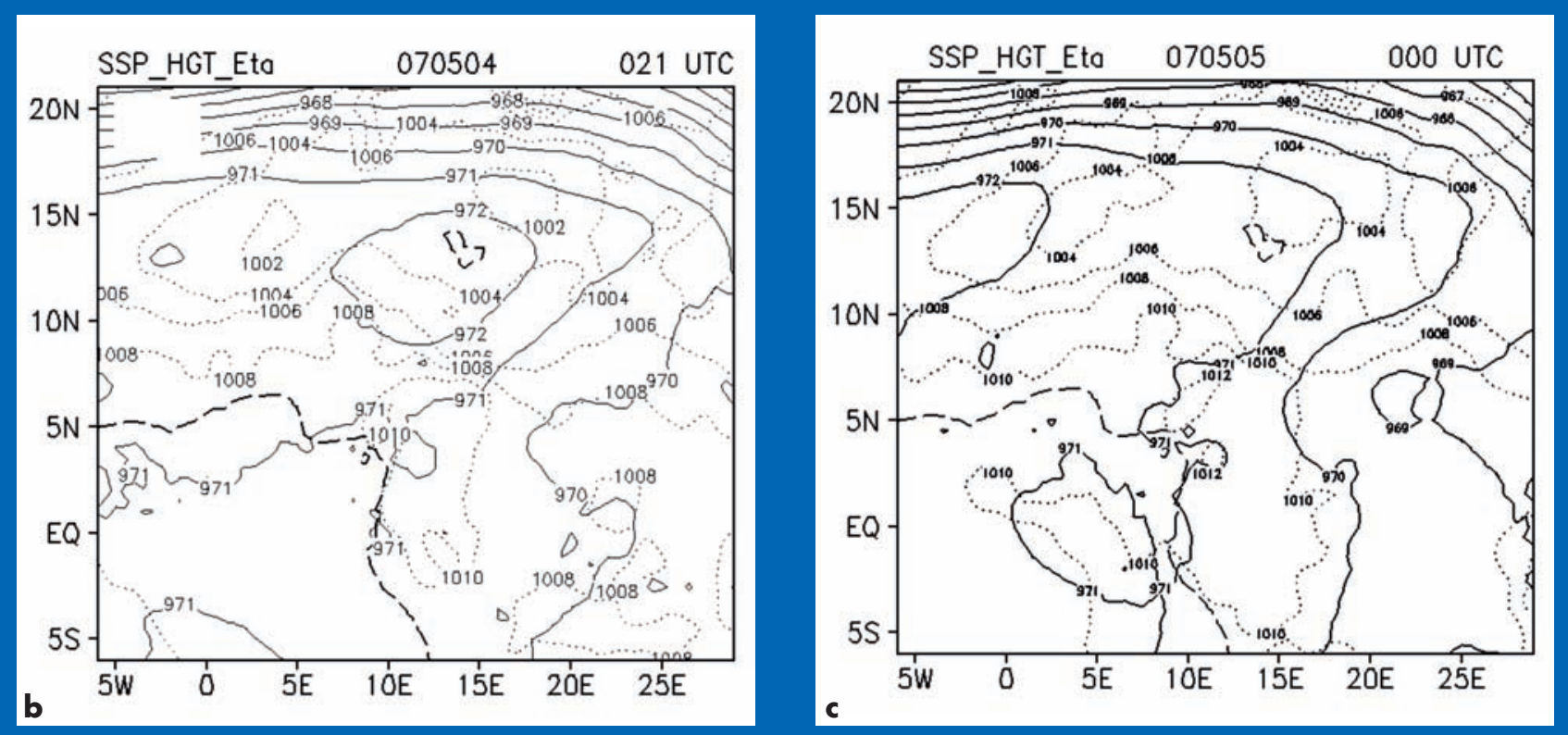

zone d'intérêt entre $21 \mathrm{~h}$ UTC du 4 mai 2007 et 03 h UTC le 5 mai 2007. Or, comme le montrent les figures précédentes, cette zone équatoriale apparaît comme étant une région d'ascendance où les flux d'énergie thermique et latente convergent. Dans cette région, les basses couches de l'atmosphère reçoivent un maximum d'énergie par le flux de chaleur sensible et par évaporation. Cela amène l'air à atteindre rapidement son niveau de condensation en s'élevant (évalué à 985 hPha à Douala ce jour-là). Cette condensation déclenche un phénomène de convection humide qui transforme l'énergie thermique et latente des basses couches en énergie potentielle pour les hautes couches. En période intense de la modulation de la mousson, on peut avoir, sur plusieurs heures, les conditions réunies pour le développement d'un violent orage, ce que confirment les prévisions (figure 14). On peut aussi noter que le modèle délimite bien la zone de précipitation, avec cependant une sous-estimation de son extension dans la zone du golfe de Guinée. On notera que les observations de TRMM font état de cumul des précipitations sur trois heures pouvant atteindre jusqu'à 100 mm à cet endroit.

\section{Conclusion}

Le caractère particulièrement remarquable du crash du Boeing B737-800, dans la nuit du 4 au 5 mai 2007 à Douala, réside, d'une part, dans le fait qu'il intervient en mai, un mois où l'activité convective est peu importante avec notamment de faibles cumuls des 


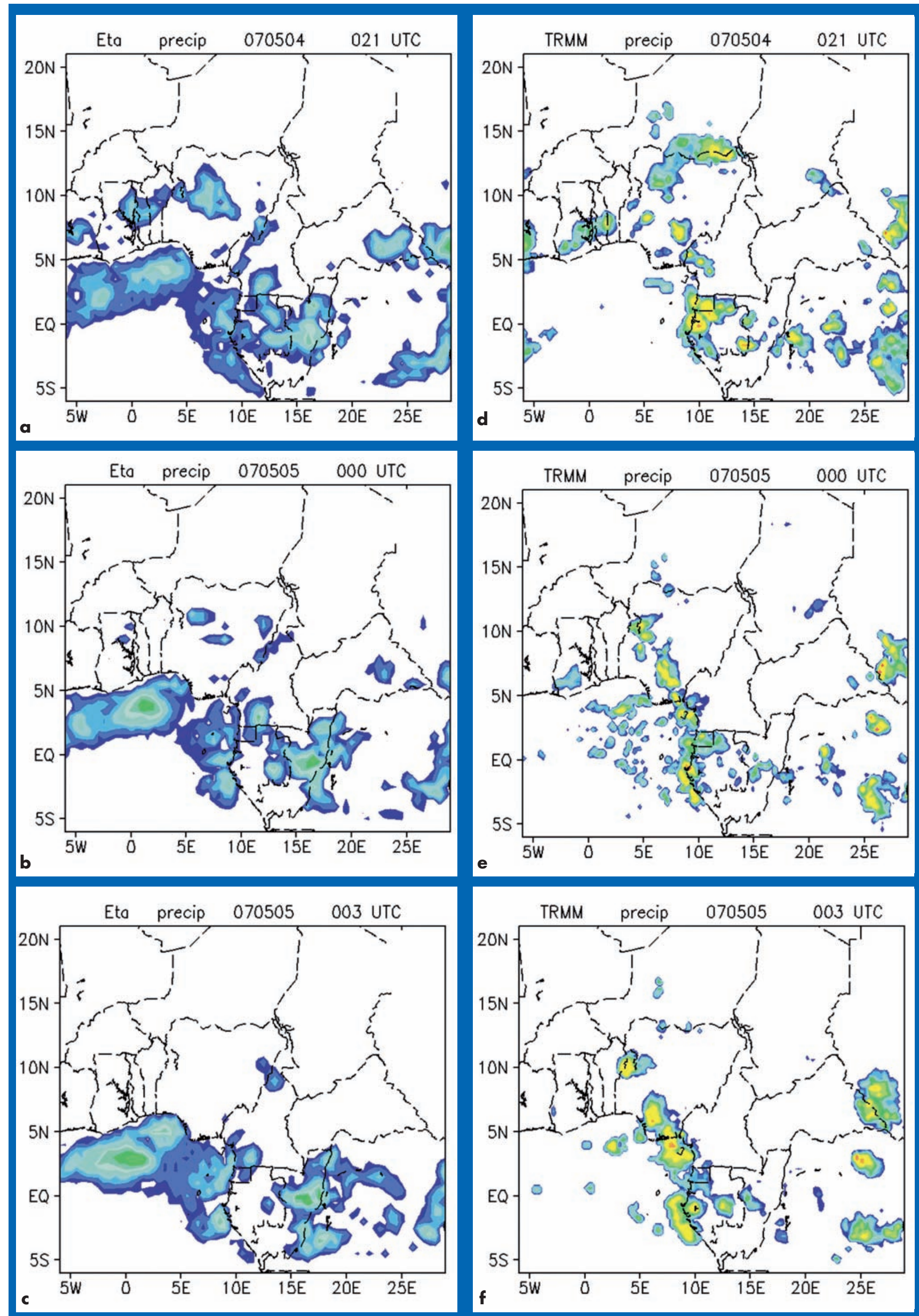

Figure 14 - Cumul sur trois heures, 18 h-21 h, 21 h-24 h, le 4 mai 2007 et de 00-03 h le 5 mai 2007, des précipitations prévues par ETA (à gauche) et issues de TRMM (à droite) autour de cette date fatidique. On voit que le modèle ETA délimite bien la zone de précipitations avec cependant une sous-estimation de son intensité par rapport au TRMM. 
précipitations, comparativement aux mois de juillet et d'août qui sont les plus pluvieux, et, d'autre part, dans le fait que le crash a lieu à six kilomè-tres seulement de la piste de décollage.

Douala est caractérisé par une climatologie particulière avec un couvert nuageux quasi permanent. Parmi ces nuages, on distingue les cumulonimbus qui sont à l'origine de la formation de la foudre. Dans cette ville, l'humidité est très forte et la chaleur très intense, ce qui conduit à de violents orages. Cela a été confirmé par l'analyse des indices de convection, avec notamment une valeur de Cape supérieure à $3500 \mathrm{~J} / \mathrm{kg}$ le 4 mai à $12 \mathrm{~h} 00$ UTC. Douala est au pied du mont Cameroun qui est à l'origine d'ascendances forcées favorisant la formation des nuages cumuliformes orageux.

La ville est aussi connue pour ses pluies intermittentes et le mois de mai se révèle être le début réel de la grande saison de pluie avec des averses qui peuvent aller jusqu'à sept jours sans interruption. L'analyse de l'image satellitaire de ce 5 mai 2007 montre que Douala est une zone orageuse avec un système convectif particulier de type $\mathrm{V}$.

En plus de l'analyse de l'image satellitaire, les conditions associées à l'intensification de la convection ont été confirmées par l'étude des champs de certains paramètres météorologiques, dont notamment la température potentielle, la vorticité potentielle et la divergence horizontale. Sur le champ de température potentielle, apparaît une masse d'air chaude au niveau de l'équateur qui se refroidit lorsque l'on se rapproche des côtes du golfe de Guinée avant de se réchauffer sur le continent. Cela est corrélé avec les variations à $925 \mathrm{hPa}$ de la vorticité et de la divergence horizontale. Cette région du golfe de Guinée est marquée par une ascendance liée à une convergence horizontale en surface au-dessous d'une zone dépressionnaire. La vorticité en surface est caractérisée par de fortes valeurs négatives, en relation avec une advection thermique chaude qui tend à renforcer l'ascendance.

Une prévision de l'événement par le modèle ETA a été faite à posteriori. De manière générale, les modèles de prévision opérationnels ont souvent sousestimé les cumuls des précipitations. Le modèle ETA utilisé dans ce travail réussit à détecter les précipitations autour de la zone d'intérêt entre $21 \mathrm{~h}$ UTC du 4 mai 2007 et 03 h UTC le 5 mai 2007. On note aussi que ce modèle délimite bien la zone de précipitation, avec cependant une sous-estimation de son extension dans la zone du golfe de
Guinée. Concernant la prévision des caractéristiques de la circulation générale, ce modèle fait apparaître une situation permanente de haute pression en surface et de dépression en altitude dans la zone du golfe de Guinée. Cela est propice au développement d'une perturbation orageuse propre à cette partie $d u$ globe.

Au terme de cette étude, il est établi que les conditions météorologiques à l'aéroport de Douala du 4 au 5 mai 2007 étaient très mauvaises pour la sécurité aérienne. Cette situation météorologique particulière mérite d'être prise en compte pour le renforcement de la sécurité aérienne.

\section{Remerciements}

Nous tenons à remercier Sébastien Freissinet, constructeur du site www.1001crash.com et du site www.dinosoria.com/climatique, qui nous a permis d'utiliser les informations tirées de ces sites. Nos sincères remerciements à NCEP/NCAR, à la division technique de l'Asecna de l'aéroport de Douala et à Météo-France pour l'acquisition des images et les données.

\section{Bibliographie}

\footnotetext{
- Colby F. P. Jr, 1984 : Convective inhibition as a predictor of convection during Ave-Sesame II. Mon. Wea. Rev., 112, $2239-2252$.

- Derek H. et J. S. Oguntoyinbo, 1987 : Climatology of West Africa. Published by Rowman \& Littlefield, 271 pages.

Ducrocq V., G. Aullo et P. Santurette, 2003 : Les précipitations intenses et les inondations des 12 et 13 novembre 1999 sur le sud de la France. La Météorologie, - 8 série, 42, 18-27.

- Fennessy M. J. et J. Shukla, 2000 : Seasonal prediction over North America with a regional model nested in a global model. J. Climate, 13, $2605-2627$.

- Franklin B., 1752 : Expériences et observations sur le tonnerre, relatives à celles de Philadelphie. Présenté par T. F. d'Alibard à l'Académie de science, le 13 mai 1752.

Freissinet S., 2007 : 1001 Crash - Statistiques accidents aériens. http://www.1001crash.com, CNIL, n 1141631c, 7 p.

Hontarrède M., 2006 : Détection et prévision du brouillard. Metmar, 213, 24-29.

International Civil Aviation Organization (ICA0), 2008 : Réglementation des services de transport aérien international. A36-WP/104/EC/14-30/8/07,

http://www.icao.int/, 9 p.

Iribarne J. V. et W. L. Godson, 1973 : Atmospheric Thermodynamics. Publié par D. Reidel Publishing Company, Dordrecht, Hollande, 222 p.

Kain J. S. et J. M. Fritsch, 1993 : Convective parameterization for mesoscale model: The Kain-Fritsch scheme. The representation of cumulus convection in numerical - models. Meteor. Monogr., 46, 165-170.

Kalnay E., M. Kanamitsu, R. Kistler, W. Collins, D. Deaven, L. Gaudin, M. Iredell, S. Saha, G. White, J. Woollen, Y. Zhu, M. Chelliah, W. Ebisuzaki, W. Higgins, J. Janowiak, K. C. Mo, C. Ropelewski, J. Wang, A. Leetmaa, R. Reynolds, R. Jenne et D. Joseph, 1996 : The NCEP/NCAR 40-year reanalysis project. Bull. Amer. Meteor. Soc., 77, 437-471.

Kerry A. E., 1994 : Atmospheric Convection. Published by Oxford University Press US, 580 p.

Mekonnen A., C. D. Thorncroft et A. R. Aiyyer, 2006 : Analysis of convection and its association with african easterly waves. J. Climate, 13, 5405-5421.

- Mesinger F., 1984 : A blocking technique for representation of mountains in atmospherics models. Riv. Meteor. Aeronaut., 44, $195-202$.

- Rogers E., 1995 : The regional analysis system for the operational ETA model: Original $80 \mathrm{~km}$ configuration and recent changes. Wea. Forecasting, 10, 810-825

- Sénési S. et R.-M. Thepenier, 1997 : Indices d'instabilité et occurrence d'orage : cas de l'Île-de-France. La Météorologie, 8e série, 19 , 18-33.

- Triplet J. P. et G. Roche, 1977 : Météorologie générale. École nationale de la météorologie, $2^{\circledR}$ édition, 317 p.

Yau M. K. et R. Rogers, 1989 : Short course in cloud physics, 3édition. Publié par Butterworth-Heinemann, 304 p.
} 